\title{
Comparison of outcomes in patients with muscle-invasive bladder cancer treated with radical cystectomy versus bladder preservation
}

\author{
Jim Zhong, MD ${ }^{1,2}$, Jeffrey Switchenko, PhD ${ }^{2,3}$, Naresh K. Jegadeesh, MD ${ }^{1,2}$, Richard J. \\ Cassidy, MD ${ }^{1,2}$, Theresa Gillespie, PhD $^{2,7}$, Viraj Master, MD $^{2,4}$, Peter Nieh, MD ${ }^{2,4}$, Mehrdad \\ Alemozaffar, MD ${ }^{2,4}$, Omer Kucuk, MD ${ }^{2,5}$, Bradley Carthon, MD ${ }^{2,5}$, Christopher P. Filson, MD, \\ MS $^{2,4,6}$, Mehmet A. Bilen, MD ${ }^{2,5}$, and Ashesh B. Jani, MD ${ }^{1,2}$ \\ ${ }^{1}$ Departments of Radiation Oncology, Emory University, Atlanta Georgia \\ ${ }^{2}$ Winship Cancer Institute, Emory University, Atlanta Georgia \\ ${ }^{3}$ Department of Biostatistics \& Bioinformatics, Emory University, Atlanta, GA \\ ${ }^{4}$ Department of Urology, Emory University \\ ${ }^{5}$ Department of Hematology and Oncology, Emory University \\ ${ }^{6}$ Atlanta Veterans Affairs Medical Center, Decatur, GA \\ ${ }^{7}$ Department of Surgery, Emory University
}

\begin{abstract}
Purpose-Radical cystectomy currently remains the standard of care for muscle-invasive bladder cancer (MIBC). However, surgery can be associated with considerable morbidity and mortality, including the removal of the bladder. An alternative strategy is to preserve the bladder through concurrent chemo-radiation following a maximal trans-urethral resection of the tumor. National protocols using a bladder preservation approach have demonstrated disease-specific outcomes comparable to radical cystectomy in selected patients, but these results have not been replicated in previously reported population-based series. Here, we describe an outcomes analysis of patients with MIBC treated with either radical surgery or bladder-preserving chemo-radiation (BPCRT) for those patients meeting BPCRT criterion using the National Cancer Database (NCDB).
\end{abstract}

\begin{abstract}
Methods and Materials-Using the NCDB, patients with AJCC clinical T2-3, N0, M0 urothelial carcinoma diagnosed between 2004-2013 were included for analysis. Only patients treated with definitive intent with either radical cystectomy or concurrent chemotherapy and radiation after a maximal transurethral tumor resection were included. Propensity-score matching was employed.
\end{abstract}

Results-Among 8,454 eligible patients, 7,276 (86\%) underwent radical cystectomy, and 1,178 (14\%) underwent BPCRT. Patients undergoing BPCRT were significantly older (median age 77 vs

Corresponding author: Jim Zhong, MD, Resident Physician, Department of Radiation Oncology, Winship Cancer Institute at Emory University, 1365 Clifton Road NE, Atlanta, GA 30322, jim.zhong@emory.edu, Phone: 404-409-5168, Fax: 404-778-3670.

Conflicts of Interest: None 
$68, \mathrm{p}<0.001)$ and had higher Charlson-Deyo comorbidity scores $(\mathrm{p}=0.002)$. Using propensitymatched analysis, 1,002 patients remained in each cohort, and there was no significant difference in survival found between the two cohorts (median OS: 2.7 vs 3.0 ( $\mathrm{p}=0.20)$; 4-year OS: $39.1 \%$ and $42.6 \%$ ( $\mathrm{p}=0.15$ ), for BPCRT and surgery, respectively). Additionally, the hazard ratio (HR) of surgery versus BPCRT decreased over time, with an initial HR of 1.27 favoring BPCRT which decreased by a factor of 0.85 per year.

Conclusions-From 2004-2013, approximately 14\% of patients from the NCDB who potentially met bladder preservation criteria underwent the procedure. Our propensity-matched analysis is the only report of its kind to demonstrate similar survival outcomes with bladder preservation when patients are properly selected. This study is also the first to demonstrate a dynamic hazard ratio between radical surgery and BPCRT over time.

\section{Keywords}

MIBC; trimodality therapy; bladder preservation therapy; bladder cancer

\section{Introduction}

Bladder cancer remains a significant clinical challenge, with over 75,000 new cases diagnosed annually[20]. The treatment of patients with muscle-invasive bladder cancers (MIBC), disease that has invaded the muscularis propria, has traditionally been managed through radical cystectomy[22]. Radical surgery, however, is accompanied by significant treatment morbidity and mortality, including the removal of the natural bladder[5]. Combined modality bladder preservation has emerged as a viable oncologic paradigm, which may allow the preservation of the natural bladder through concurrent chemotherapy and radiation after aggressive transurethral tumor resection in select patients[3,6,13,15]. Similar approaches for organ preservation using definitive chemo-radiation in lieu of radical resection have been adopted for selected stages in other disease sites such as cancers of the oropharynx, larynx, and anal canal.

Over the past three decades, the Radiation Therapy Oncology Group (RTOG) and British Columbia (BC) Cooperative Groups have conducted several multicenter studies examining the outcomes of bladder preservation with chemoradiotherapy[8-11,19,24]. Recent reports of pooled analysis with 10-year outcomes in these patients demonstrated comparable survival outcomes to those of radical surgery[6,13,22]. Additionally, the rate of bladderintact disease-free survival has been reported to be 50-60\% at 10 years. Despite these promising reports, bladder-preserving strategies through the use of concurrent chemoradiation have not been adopted as a standard therapy.

These previously reported series investigating bladder-preserving chemo-radiation (BPCRT) represent carefully selected patients treated within strict trial protocols. Thus, the outcomes of these clinical protocols may differ from those achieved in routine clinical practice. Here we utilize the National Cancer Database (NCDB) to directly compare the outcomes of patients with MIBC treated with radical cystectomy with BPCRT in a real-world practice setting. 


\section{Methods and Materials}

\section{Patient Cohorts}

The National Cancer Database (NCDB) registry was used to identify study subjects. The NCDB is a national clinical oncology database sourced from hospital registry data collected from over 1,500 Commission on Cancer ( $\mathrm{CoC})$-accredited facilities and captures at least $70 \%$ of new cancer cases in the United States [14,17]. The NCDB contains valuable information not available in other national registries, including detailed radiotherapy information. Data is reported retrospectively to the NCDB and contains no patient or physician identifiers and is consequently exempt from the (blinded) Institutional Review Board (IRB).

Patients with a first and only diagnosis of pathologically confirmed urothelial carcinoma were queried from the NCDB. Patients with American Joint Commission on Cancer (AJCC) clinically staged T-stage 2 and 3, $\mathrm{N}$-stage 0 , and $\mathrm{M}$-stage 0 were included for analysis, as these patients would have qualified for previous national bladder preservation studies $[8,10,13,19]$. Patients documented to have undergone radical cystectomy or anterior exenteration was included in the surgery cohort. Patients who underwent radiation for initial treatment were excluded from the surgery cohort. Only patients documented to have received a trans-urethral tumor resection (TURBT) and external beam irradiation to the pelvis and/or bladder with greater than 25 total radiation fractions with single or multi-agent chemotherapy were included in the BPCRT cohort. Patients receiving palliative surgery or radiation were excluded.

\section{Statistical analyses}

Descriptive statistics were compiled to summarize the patient demographics, disease and treatment characteristics. Association of the baseline characteristics with treatment group was assessed using chi-squared tests or ANOVA, where appropriate. For the surgery cohort, overall survival (OS) was calculated from first treatment date (either definitive surgery or receipt of chemotherapy) to date of death or last follow-up. For the BPCRT cohort, OS was calculated from first treatment date (either receipt of chemotherapy or radiation therapy) to date of death or last follow-up. As such, if a patient received neoadjuvant chemotherapy, his/her survival is calculated from the start of the neoadjuvant chemotherapy for both cohorts. Univariate association of patient, tumor, and treatment factors with OS was evaluated using log-rank tests, and OS were estimated using the Kaplan-Meier (KM) method. Yearly KM rates between treatment groups also were compared using Z-tests. Univariate (UV) and multivariable (MV) Cox proportional hazards (PH) models were fit for OS as a function of patient demographics, disease and treatment characteristics. In the MV model, the presence of multicollinearity was checked via variance inflation factors. The proportional hazards assumption was also checked. In cases where the PH assumption was violated, and an interaction was fit between the covariate and overall survival, and a timevarying hazard ratio was reported. Statistical analyses were performed using SAS version 9.3 (Cary, NC), and SAS macros or software developed at the Biostatistics and Bioinformatics Shared Core Resource at Emory University [16]. 
Propensity score analysis was also performed to reduce treatment selection bias, utilizing the propensity score matching method. Covariates used for propensity matching are ones calculated to significantly affect OS on MV analysis and included: patient age, facility type, Charlson-Deyo comorbidity score, disease site, and AJCC T-stage. Patients from each cohort were matched at a ratio of 1:1 based on the propensity score using a 5-1 digit match algorithm. The balance of the propensity matching between cohorts was assessed by their standardized differences, with a value of $<0.1$ to be considered a negligible difference[2].

\section{Results}

Between 2004-2013, there were 439,188 cases of bladder cancer in the NCDB queried for this study. Of these patients, 7,276 and 1,178 patients met our criteria for definitive treatment with radical cystectomy and BPCRT, respectively (Fig 1). The time from diagnosis to the first day of treatment was similar for both cohorts, with a median of 1.5 months [25th75th percentile: $0.9-2.3$ months] and 1.5 months [25th-75th percentile: $1.0-2.4$ months], for the BPCRT and surgery cohorts, respectively. At baseline, there were significant differences between the groups in several patient and tumor characteristics (Table 1). Patients who were treated with BPCRT were older (median age: 75 for BPCRT vs 67 for surgery), had more medical comorbidities (Charlson-Deyo Score 0: 65.6\% for BPCRT vs $69.5 \%$ for surgery), and lived closer to their treatment center (median miles: 7.5 BPCRT for and 16.4 for surgery), all $\mathrm{p}<0.001$. Patients treated with BPCRT had a slightly lower proportion of clinically staged $\mathrm{T} 3$ tumors ( $11.3 \%$ for BPCRT and $13.5 \%$ for surgery, $\mathrm{p}=0.036$ ). The primary site of disease in the bladder also differed between the two cohorts $(\mathrm{p}<0.001)$. Kaplan-Meier curves for the two cohorts are displayed in Figure 2a, demonstrating median OS of 2.6 and 3.8 years for the BPCRT and surgery cohorts, respectively $(\mathrm{p}<0.001)$.

On multivariable analysis, advanced age at diagnosis, advanced clinical T-stage, and higher Charlson-Deyo comorbidity score were all found to be unfavorably associated with overall survival (all $\mathrm{p}<0.001$ ) (Table 3). Primary disease located in the bladder wall, neck, and ureteric orifice were found to be favorably associated with survival $(p<0.001)$. Treatment at academic/research programs was associated with favorable OS compared with comprehensive community $(\mathrm{p}<0.001)$ and community cancer programs $(\mathrm{p}<0.007)$. The hazard ratio for treatment with surgery vs. bladder preservation was found to be dependent on time, with an initial HR of 1.27 (95\% Confidence Interval (CI): 1.11-1.44) favoring the CRT cohort and decreases by a factor of 0.85 (95\% CI: 0.81-0.89) per year for surviving patients. The time-varying HR between surgery and CRT over time is depicted in Figure 3.

\section{Propensity-matched analysis}

Patients were propensity-matched based on age at diagnosis, treatment facility type, Charlson-Deyo comorbidity index, primary disease site within the bladder, and clinical Tstaging. The median follow-up time was 4.5 years for all patients. In the BPCRT cohort, 529 (52.8\%) patients received single-agent chemotherapy, 391 (39.0\%) patients received multiagent chemotherapy, and $82(8.2 \%)$ patients received unspecified chemotherapy. The radiation therapy administered was exclusively external beam irradiation with photons. The median radiation dose received was $45 \mathrm{~Gy}$ in 25 fractions with an additional median boost of 
19.8 Gy. The median total radiation dose was $64.8 \mathrm{~Gy}$ [10th-90th percentile: 56.0-66.6 Gy], and the median total number of fractions in the CRT was 36 [10th-90th percentile: 30-37]. In the propensity-matched surgical cohort, 309 (30.8\%) patients received chemotherapy, with 107 (10.7\%) receiving neoadjuvant, 144 (14.4\%) undergoing adjuvant, and 20 (2.0\%) undergoing both neoadjuvant and adjuvant chemotherapy. Of those undergoing surgery, 854 $(85.2 \%)$ patients were reported to have negative resection margins, $96(9.6 \%)$ with involved margins, and $52(5.2 \%)$ with unknown margin status. Following propensity matching, 1,002 patients were assigned to each cohort, and the patient and disease characteristics were well matched with the exception of facility type, limited by the patient size in these subgroups (Table 2).

The Kaplan-Meier curves for the propensity-matched cohorts are displayed in Figure $2 \mathrm{~b}$ and demonstrate similar median survival of 2.7 (95\% CI: $2.4-3$ ) and 3.0 years (95\% CI: $2.6-3.4$ ) for the BPCRT and surgery cohorts, respectively $(\mathrm{p}=0.20)$. Likewise, the 4-year OS for the BPCRT and surgery cohorts were not statistically different at $39.1 \%$ and $42.6 \%$, respectively $(\mathrm{p}=0.15)$. Due to a violation of the proportional hazards assumption, an interaction between treatment group and overall survival was fit for the propensity matched groups, and a timevarying hazard ratio was produced. It demonstrated that the log hazard ratio of surgery vs. BPCRT reduced by 0.16 (95\% CI: $0.11-0.19)$ for every year increase in survival time (Figure 3).

\section{Discussion}

The objective of this study was to compare survival outcomes in patients with clinicallystaged T2-T3N0M0 MIBC treated in the United States with either radical surgery or BPCRT. Our findings demonstrate similar median overall survival times in patients with MIBC treated with these two treatment strategies when propensity-matched. These results suggest that not only is BPCRT feasible outside the clinical trial setting but also that the outcomes are comparable to those of radical cystectomy when patients are propensitymatched. Our data additionally confirms patient and disease characteristics previously reported to be negatively associated with survival, such as advanced age, advanced tumor stage, and poor baseline performance status[6,7].

Our study is the first to suggest a demonstrable change in hazard ratio between receipt of radical surgery versus BPCRT. The finding of a time-varying hazard ratio between the two treatment groups may be accounted for in several ways. It may be that despite propensity matching, there still exists confounding factors leading to deaths unrelated to cancer in the BPCRT cohort, given the lack of granularity of the Charlson-Deyo score as a measure of performance status[12]. It is also possible that those patients treated with surgery initially had additional salvage treatment options unavailable to those in the BPCRT group. Additionally, it has been documented that surgery-related deaths most commonly occur within the first 3 months following surgery, which could account for the initially low survival rate seen in this cohort[23]. Unfortunately, the NCDB does not provide detailed data regarding cause-specific death, and without such data, this finding is purely hypothesis generating at this time. Seisen et al. recently published a comparative study using the NCDB suggesting that long-term survivors treated with trimodality therapy were more likely to die 
than those treated with radical surgery [18]. The time-varying hazard ratio from our data reconciles the outcomes of these two reports suggesting that at follow-up periods greater than five years, those patients in the surgery cohort who are still alive have a higher probability of surviving in the future. Our series also excluded those patients with T4 tumors, as these patients were generally excluded from bladder-preservation studies. Thus, the discrepancies in these two studies may also be due to better selection of patients suitable for bladder-preserving strategy.

The overall survival rates for both the BPCRT and surgical cohorts in this series were lower than those previously reported. The clinical trials utilizing BPCRT for patients with MIBC by the RTOG and BC cooperative groups demonstrated 5-year overall survival rates between $35 \%-83 \%[6,8,10,11,15,19]$. Similarly, large series investigating cystectomy outcomes with or without neoadjuvant chemotherapy have been reported to be $45 \%-60 \%[1,4,7]$. The lower survival rates in our series for both treatment cohorts is likely multifactorial. In our BPCRT cohort, approximately $30 \%$ of patients had significant medical comorbidities, with CharlsonDeyo Scores of 1 or 2, which may have excluded them from participation in the cooperative group trials. Additionally, treatment outside the strictly regulated clinical trial setting may allow patients to receive sub-optimal doses/fields of radiation, chemotherapy, or both deviating from trial protocol.

Likewise, previously published cystectomy outcomes represent data from select highvolume centers, which has been associated with better cancer-specific survival and overall survival[21]. In our series, approximately $30 \%$ of patients were not treated at academic/ research programs or comprehensive cancer programs. Additionally, while a survival benefit has been demonstrated on meta-analysis with the addition of neoadjuvant chemotherapy, only $30 \%$ of patients in our surgical cohort received systemic therapy, further accounting for inferior results[7]. Despite the lower survival rates presented in this series for both treatment strategies, our data represents actual outcomes in patients treated across the country.

Our analysis has several limitations. This study is a retrospective analysis of a national oncologic registry and carries limitations inherent in all retrospective studies. Namely, implied selection bias would be expected in such a study which seeks to retrospectively compare treatment with a curative surgery with a nonsurgical alternative. Given these considerations, we have taken every possible step to propensity-match our cohorts with factors known to be associated with surgical in this patient population. The NCDB registry also lacks data regarding local control, treatment-related morbidity, chemotherapy agent, and specific cause of death. Additionally, patients who may have received salvage radical cystectomy following CRT are not accurately captured and are not reported in our series. Moreover, detailed information regarding the chemotherapy agent, dose, and duration of treatment is not recorded by the NCDB. Furthermore, the NCDB does not capture toxicity data, and we believe that long-term toxicity from either treatment as well as patient-related outcomes could be crucial in the multi-disciplinary decision making for patients with this disease. These factors should be taken into account for any future prospective studies.

Despite these limitations, this study contains a significant number of patients and directly compares the outcomes of patients with MIBC treated with definitive surgery versus 
bladder-preserving chemoradiation. Our results suggest that patients can be treated with the bladder-preserving approach outside the clinical trial setting with results comparable to those of radical surgery. Additionally, our series is the only large series of patients to show equivalent survival when patients suitable for bladder-preserving strategy are properly selected. In the absence of prospective, multi-center randomized trials, this study may help guide treatment decision in the multidisciplinary setting.

\section{Acknowledgments}

Research reported in this publication was supported in part by the Biostatistics and Bioinformatics Shared Resource of Winship Cancer Institute of Emory University and NIH/NCI under award number P30CA138292. The content is solely the responsibility of the authors and does not necessarily represent the official views of the National Institutes of Health.

\section{References}

1. Advanced Bladder Cancer Meta-analysis C. Neoadjuvant chemotherapy in invasive bladder cancer: Update of a systematic review and meta-analysis of individual patient data advanced bladder cancer (abc) meta-analysis collaboration. Eur Urol. 2005; 48:202-205. [PubMed: 15939524]

2. Austin PC, Grootendorst P, Anderson GM. A comparison of the ability of different propensity score models to balance measured variables between treated and untreated subjects: A monte carlo study. Stat Med. 2007; 26:734-753. [PubMed: 16708349]

3. Chen RC, et al. Trimodality bladder preservation therapy for muscle-invasive bladder cancer. J Natl Compr Canc Netw. 2013; 11:952-960. [PubMed: 23946174]

4. Clark PE, et al. Radical cystectomy in the elderly: Comparison of survival between younger and older patients. Cancer. 2005; 103:546-552. [PubMed: 15630702]

5. Dhar NB, et al. Outcome after radical cystectomy with limited or extended pelvic lymph node dissection. J Urol. 2008; 179:873-878. [PubMed: 18221953]

6. Efstathiou JA, et al. Long-term outcomes of selective bladder preservation by combined-modality therapy for invasive bladder cancer: The mgh experience. Eur Urol. 2012; 61:705-711. [PubMed: 22101114]

7. Grossman HB, et al. Neoadjuvant chemotherapy plus cystectomy compared with cystectomy alone for locally advanced bladder cancer. N Engl J Med. 2003; 349:859-866. [PubMed: 12944571]

8. Hagan MP, et al. Rtog 97-06: Initial report of a phase i-ii trial of selective bladder conservation using turbt, twice-daily accelerated irradiation sensitized with cisplatin, and adjuvant mcv combination chemotherapy. Int J Radiat Oncol Biol Phys. 2003; 57:665-672. [PubMed: 14529770]

9. James ND, et al. Radiotherapy with or without chemotherapy in muscle-invasive bladder cancer. N Engl J Med. 2012; 366:1477-1488. [PubMed: 22512481]

10. Kaufman DS, et al. The initial results in muscle-invading bladder cancer of rtog 95-06: Phase i/ii trial of transurethral surgery plus radiation therapy with concurrent cisplatin and 5-fluorouracil followed by selective bladder preservation or cystectomy depending on the initial response. Oncologist. 2000; 5:471-476. [PubMed: 11110598]

11. Kaufman DS, et al. Phase i-ii rtog study (99-06) of patients with muscle-invasive bladder cancer undergoing transurethral surgery, paclitaxel, cisplatin, and twice-daily radiotherapy followed by selective bladder preservation or radical cystectomy and adjuvant chemotherapy. Urology. 2009; 73:833-837. [PubMed: 19100600]

12. Lin CC, et al. Comparison of comorbid medical conditions in the national cancer database and the seer-medicare database. Ann Surg Oncol. 2016

13. Mak RH, et al. Long-term outcomes in patients with muscle-invasive bladder cancer after selective bladder-preserving combined-modality therapy: A pooled analysis of radiation therapy oncology group protocols 8802, 8903, 9506, 9706, 9906, and 0233. J Clin Oncol. 2014; 32:3801-3809.

[PubMed: 25366678] 
14. Menck HR, et al. The growth and maturation of the national cancer data base. Cancer. 1997; 80:2296-2304. [PubMed: 9404707]

15. Mitin T, et al. Long-term outcomes among patients who achieve complete or near-complete responses after the induction phase of bladder-preserving combined-modality therapy for muscleinvasive bladder cancer: A pooled analysis of nrg oncology/rtog 9906 and 0233. Int J Radiat Oncol Biol Phys. 2016; 94:67-74. [PubMed: 26700703]

16. (BLINDED).

17. Partridge EE. The national cancer data base: Ten years of growth and commitment. CA Cancer $\mathbf{J}$ Clin. 1998; 48:131-133. [PubMed: 9594915]

18. Seisen T, et al. Comparative effectiveness of trimodal therapy versus radical cystectomy for localized muscle-invasive urothelial carcinoma of the bladder. Eur Urol. 2017

19. Shipley WU, et al. Phase iii trial of neoadjuvant chemotherapy in patients with invasive bladder cancer treated with selective bladder preservation by combined radiation therapy and chemotherapy: Initial results of radiation therapy oncology group 89-03. J Clin Oncol. 1998; 16:3576-3583. [PubMed: 9817278]

20. Siegel R, Naishadham D, Jemal A. Cancer statistics, 2013. CA Cancer J Clin. 2013; 63:11-30. [PubMed: 23335087]

21. Siemens DR, et al. Processes of care and the impact of surgical volumes on cancer-specific survival: A population-based study in bladder cancer. Urology. 2014; 84:1049-1057. [PubMed: 25443899]

22. Stein JP, et al. Radical cystectomy in the treatment of invasive bladder cancer: Long-term results in 1,054 patients. J Clin Oncol. 2001; 19:666-675. [PubMed: 11157016]

23. Tang K, et al. Laparoscopic versus open radical cystectomy in bladder cancer: A systematic review and meta-analysis of comparative studies. PLoS One. 2014; 9:e95667. [PubMed: 24835573]

24. Tester W, et al. Neoadjuvant combined modality program with selective organ preservation for invasive bladder cancer: Results of radiation therapy oncology group phase ii trial 8802. J Clin Oncol. 1996; 14:119-126. [PubMed: 8558186] 
439,188 NCDB bladder cancer cases

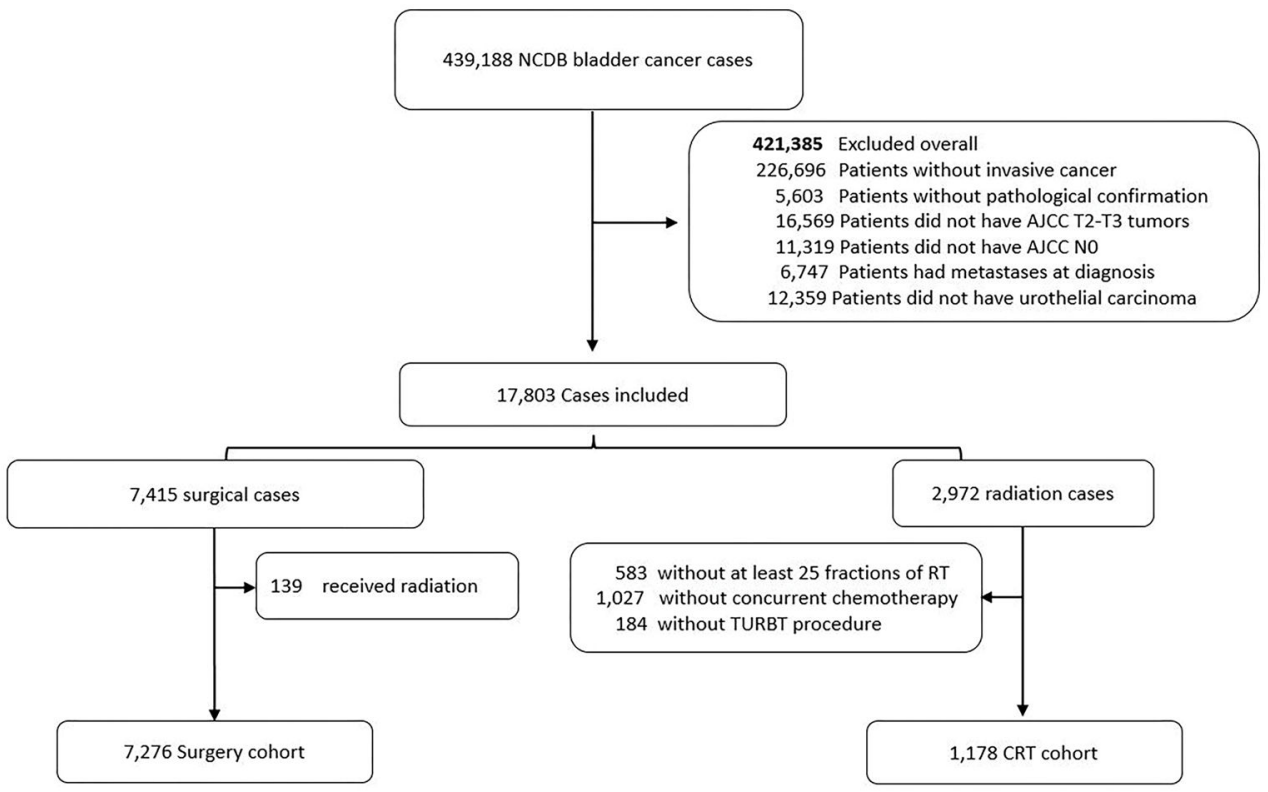

Figure 1.

Patient consort diagram

Am J Clin Oncol. Author manuscript; available in PMC 2020 January 01. 

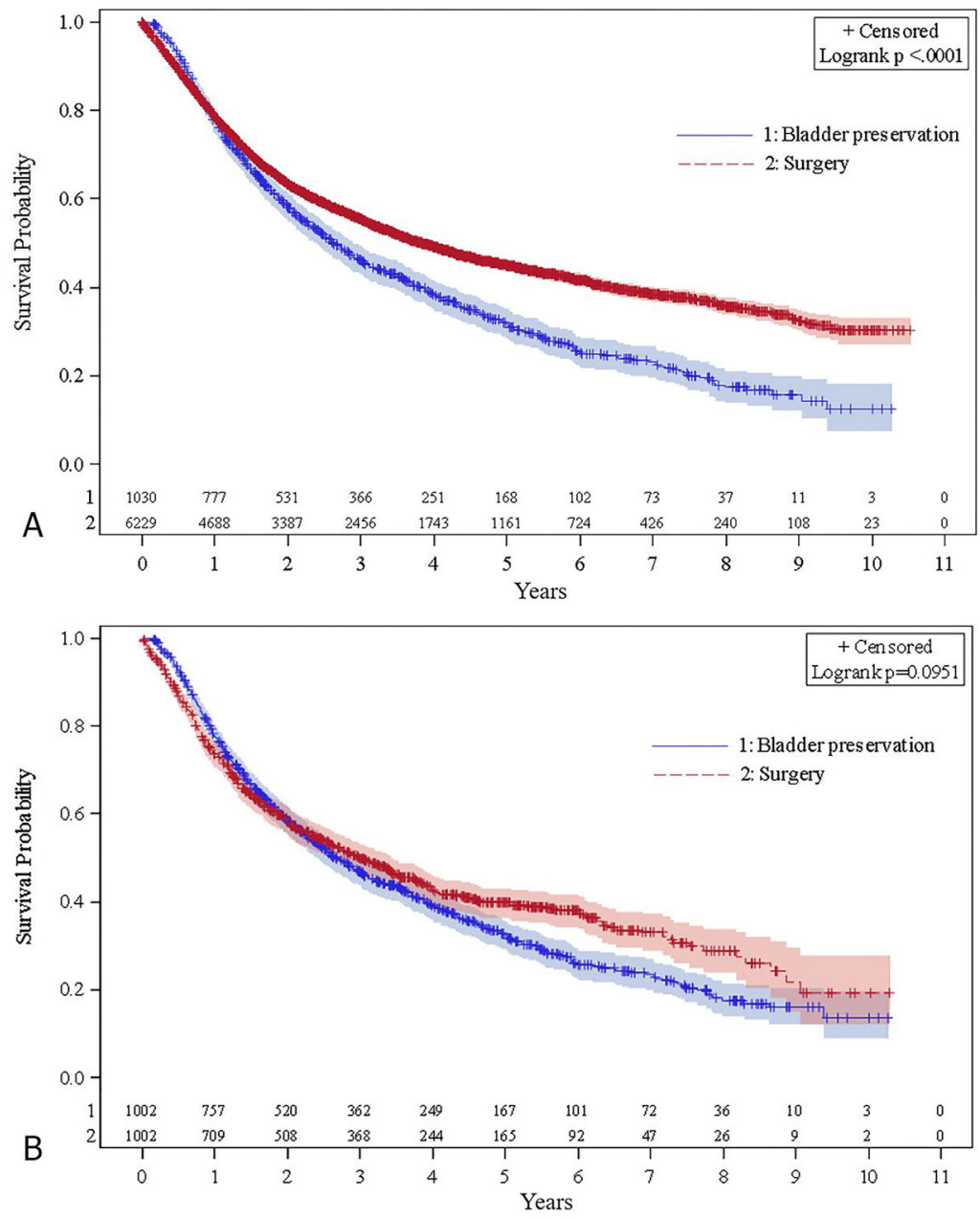

Figure 2.

Kaplan Meier curves demonstrating overall survival for unmatched cohorts (A) and propensity-matched cohorts (B) 


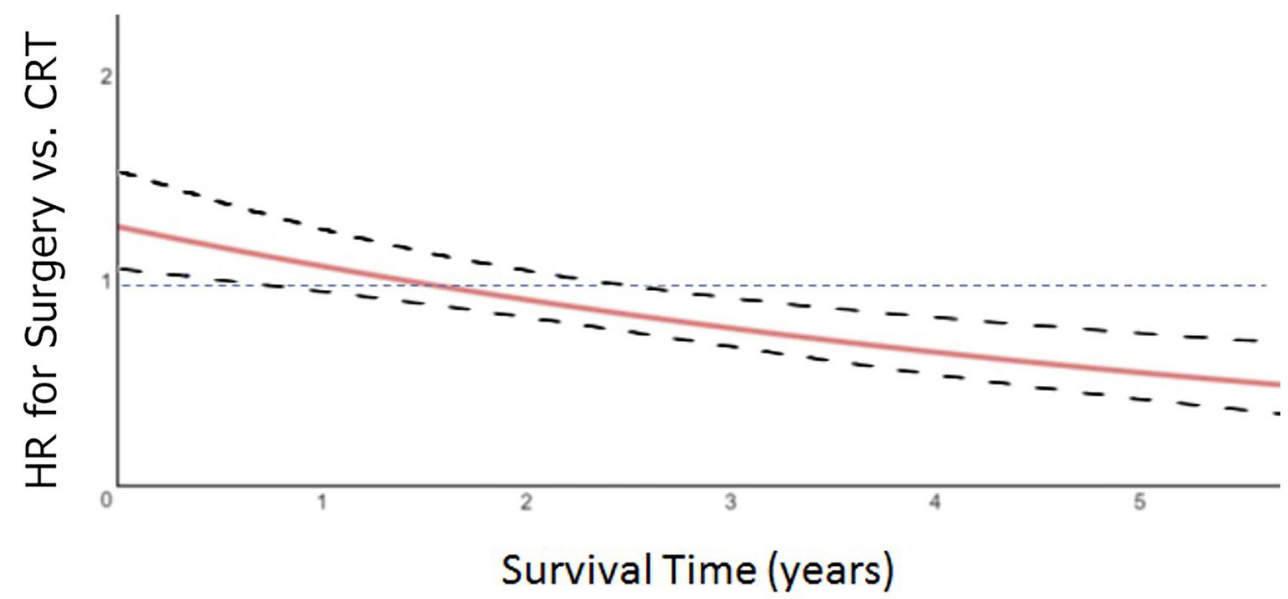

Figure 3.

Hazard ratio for surgery cohort vs CRT as a function of survival years (solid line). Dotted lines represent the $95 \%$ confidence interval. 


\section{Table 1}

Baseline patient demographics and tumor characteristics for surgery and BPCRT cohorts

\begin{tabular}{|c|c|c|c|c|}
\hline & & Surgery $(n=7,276)$ & Bladder preservation $(\mathrm{n}=\mathbf{1 , 1 7 8})$ & P-value \\
\hline $\begin{array}{l}\text { Age at Diagnosis } \\
\text { (median) }\end{array}$ & & 67.39 & 75.21 & $<.001$ \\
\hline \multirow[t]{2}{*}{ Sex } & Male & $5499(75.58)$ & $863(73.26)$ & \multirow[t]{2}{*}{0.087} \\
\hline & Female & $1777(24.42)$ & $315(26.74)$ & \\
\hline \multirow[t]{5}{*}{ Treatment Facility Type } & Community Cancer Program & $531(7.33)$ & $170(14.44)$ & $<.001$ \\
\hline & $\begin{array}{c}\text { Comprehensive Community Cancer } \\
\text { Program }\end{array}$ & $2648(36.56)$ & $617(52.42)$ & \\
\hline & Academic/Research Program & $3591(49.59)$ & $303(25.74)$ & \\
\hline & Integrated Network Cancer Program & $438(6.05)$ & $86(7.31)$ & \\
\hline & Other specified types of cancer programs & $34(0.47)$ & $1(0.08)$ & \\
\hline \multirow[t]{3}{*}{ Charlson-Deyo Score } & 0 & $5054(69.46)$ & $773(65.62)$ & \multirow[t]{3}{*}{0.002} \\
\hline & 1 & $1722(23.67)$ & $294(24.96)$ & \\
\hline & 2 & $500(6.87)$ & $111(9.42)$ & \\
\hline \multirow[t]{5}{*}{ Primary site } & Trigone of bladder & $444(6.1)$ & $71(6.03)$ & \multirow[t]{5}{*}{$<.001$} \\
\hline & Dome of bladder & $258(3.55)$ & $55(4.67)$ & \\
\hline & Bladder wall & $2016(27.71)$ & $360(30.56)$ & \\
\hline & Bladder neck and ureteric orifice & $237(3.26)$ & $62(5.26)$ & \\
\hline & Bladder NOS & $4321(59.39)$ & $630(53.48)$ & \\
\hline \multirow[t]{2}{*}{ Grade } & Well differentiated/Moderately differentiated & $196(2.98)$ & $42(3.98)$ & \multirow[t]{2}{*}{0.083} \\
\hline & Poorly differentiated/Undifferentiated & $6371(97.02)$ & $1012(96.02)$ & \\
\hline \multirow[t]{2}{*}{ T-stage } & 2 & $6292(86.48)$ & $1045(88.71)$ & \multirow[t]{2}{*}{0.036} \\
\hline & 3 & $984(13.52)$ & $133(11.29)$ & \\
\hline $\begin{array}{l}\text { Distance from } \\
\text { treatment center } \\
\text { (median miles) }\end{array}$ & & 16.4 & 7.5 & $<.001$ \\
\hline
\end{tabular}




\section{Table 2}

Baseline patient demographics and tumor characteristics for propensity-matched cohorts

\begin{tabular}{|c|c|c|c|c|}
\hline & & Surgery $(n=1002)$ & Bladder preservation $(\mathrm{n}=1002)$ & Standardized Difference \\
\hline $\begin{array}{l}\text { Age at Diagnosis } \\
\text { (median) }\end{array}$ & & $73.95(9.58)$ & $74.81(9.48)$ & 0.090 \\
\hline \multirow[t]{5}{*}{ Facility Type } & Community Cancer Program & $112(11.18)$ & $133(13.27)$ & 0.064 \\
\hline & $\begin{array}{l}\text { Comprehensive Community } \\
\text { Cancer Program }\end{array}$ & $547(54.59)$ & $529(52.79)$ & 0.036 \\
\hline & Academic/Research Program & $173(17.27)$ & $265(26.45)$ & 0.224 \\
\hline & $\begin{array}{l}\text { Integrated Network Cancer } \\
\text { Program }\end{array}$ & $169(16.87)$ & $74(7.39)$ & 0.294 \\
\hline & $\begin{array}{l}\text { Other specified types of } \\
\text { cancer programs }\end{array}$ & $1(0.1)$ & $1(0.1)$ & 0.000 \\
\hline \multirow[t]{3}{*}{ Charlson-Deyo Score } & 0 & $668(66.67)$ & $658(65.67)$ & 0.021 \\
\hline & 1 & $244(24.35)$ & $254(25.35)$ & 0.023 \\
\hline & 2 & $90(8.98)$ & $90(8.98)$ & 0.000 \\
\hline \multirow[t]{5}{*}{ Primary site } & Trigone of bladder & $51(5.09)$ & $61(6.09)$ & 0.043 \\
\hline & Dome of bladder & $44(4.39)$ & $45(4.49)$ & 0.005 \\
\hline & Bladder wall & $320(31.94)$ & $300(29.94)$ & 0.043 \\
\hline & $\begin{array}{l}\text { Bladder neck and ureteric } \\
\text { orifice }\end{array}$ & $44(4.39)$ & $49(4.89)$ & 0.024 \\
\hline & Bladder NOS & $543(54.19)$ & $547(54.59)$ & 0.008 \\
\hline \multirow[t]{2}{*}{ T-stage } & 2 & $910(90.82)$ & $886(88.42)$ & 0.079 \\
\hline & 3 & $92(9.18)$ & $116(11.58)$ & 0.079 \\
\hline
\end{tabular}


Table 3

Multivariable analysis for the association between patient and tumor characteristics with overall survival

\begin{tabular}{|c|c|c|c|}
\hline & & Hazard Ratio & HR P-value \\
\hline \multirow[t]{2}{*}{ Treatment group } & Surgery & $1.27(1.11-1.44)^{*}$ & $<0.001$ \\
\hline & Bladder preservation & - & - \\
\hline Age at Diagnosis & & $1.03(1.02-1.03)$ & $<.001$ \\
\hline \multirow[t]{2}{*}{ T-stage } & 2 & $0.78(0.71-0.85)$ & $<.001$ \\
\hline & 3 & - & - \\
\hline \multirow[t]{3}{*}{ Charlson-Deyo Score } & 0 & $0.61(0.55-0.68)$ & $<.001$ \\
\hline & 1 & $0.77(0.69-0.87)$ & $<.001$ \\
\hline & 2 & - & - \\
\hline \multirow[t]{5}{*}{ Primary site } & Trigone of bladder & $0.90(0.79-1.04)$ & 0.146 \\
\hline & Dome of bladder & $1.02(0.86-1.20)$ & 0.810 \\
\hline & Bladder wall & $0.80(0.74-0.86)$ & $<.001$ \\
\hline & Bladder neck and ureteric orifice & $0.69(0.57-0.83)$ & $<.001$ \\
\hline & Bladder NOS & - & - \\
\hline \multirow[t]{5}{*}{ Facility Type } & Academic/Research Program & - & - \\
\hline & Comprehensive Community Cancer Program & $1.15(1.07-1.23)$ & $<.001$ \\
\hline & Integrated Network Cancer Program & $1.13(0.99-1.30)$ & 0.067 \\
\hline & Other specified types of cancer programs & $1.19(0.69-2.06)$ & 0.528 \\
\hline & Community Cancer Program & $1.18(1.04-1.32)$ & 0.007 \\
\hline
\end{tabular}

* Hazard ratio for surgery vs. CRT is initially significant favoring CRT and decreases by a factor of 0.85 (95\% CI: $0.81-0.89)$ per year for surviving patients 